\title{
Mechanical, Structural, and Dynamical Modifications of Cholesterol Exposed Porcine Aortic Elastin
}

\author{
Kubra Bilici, ${ }^{\dagger}$ Steven W. Morgan, ${ }^{\ddagger}$ Moshe C. Silverstein, ${ }^{\dagger}$ Yunjie Wang, ${ }^{\top}$ Hyung Jin \\ Sun, "Katherine Zhang," and Gregory S. Boutis*,†,§ \\ Department of Physics, Brooklyn College, The City University of New York, 2900 Bedford \\ Avenue, Brooklyn NY, Division of Science and Mathematics, University of Minnesota, \\ Morris, 600 E 4th St Moris, MN, Department of Mechanical Engineering and Department \\ of Biomedical Engineering, Boston University, 110 Cummington Mall, Boston MA, and \\ Department of Physics, , The Graduate Center of The City University of New York, 365 \\ 5th Ave, New York, NY \\ E-mail: gboutis@brooklyn.cuny.edu
}

\begin{abstract}
Elastin is a protein of the extracellular matrix that contributes significantly to the elasticity of connective tissues. In this study, we examine dynamical and structural modifications of aortic elastin exposed to cholesterol by NMR spectroscopic and relaxation methodologies. Macroscopic measurements are also presented and reveal that cholesterol treatment may cause a decrease in the stiffness of tissue. ${ }^{2} \mathrm{H}$ NMR relaxation techniques revealed differences between the relative populations of water that

${ }^{*}$ To whom correspondence should be addressed

${ }^{\dagger}$ Brooklyn College of CUNY

$\ddagger$ University of Minnesota, Morris

"Boston University

${ }^{\S}$ The Graduate Center of The City University of New York
\end{abstract}


correlate with the swelling of the tissue following cholesterol exposure. ${ }^{13} \mathrm{C}$ magic-anglespinning NMR spectroscopy and relaxation methods indicate that cholesterol treated aortic elastin appears more mobile than control samples. Molecular dynamics simulations on a short elastin repeat VPGVG in the presence of cholesterol are used to investigate the energetic and entropic contributions to the retractive force, in comparison to the same peptide in water. Peptide stiffness is observed to reduce following cholesterol exposure due to a decrease in the entropic force.

Keywords: Elastin, ${ }^{13} \mathrm{C}$ MAS NMR, ${ }^{2} \mathrm{H}$ NMR, Cholesterol

\section{Introduction}

Elastin is a protein in the extracellular matrix that provides mechanical properties of elasticity and extensibility to connective tissues, including arteries ${ }^{1}$. These characteristics allow arteries to accommodate deformations encountered during physiological functions and maintain a steady pressure for regular blood flow throughout the body ${ }^{2,3}$. Elastin contains hydrophobic domains, which makes it attractive for interactions with ligands such as cholesterol $^{4}$. The lipid content of vascular arteries includes cholesterol and is known to increase with age and with various pathological conditions ${ }^{5,6}$, which may lead to arterial stiffening ${ }^{7,8}$. Biochemical analyses of arterial elastin (from human atherosclerotic subjects) have shown that the lipids accumulating on elastic membranes of atherosclerotic lesions are associated with elastin even in early atherosclerosis. In mild and moderate atherosclerosis changes in elastin are limited to the plaque areas of the arterial intimae while the composition of elastin

in the adjoining intimae and midiae were observed to remain unaltered. However, in severe atherosclerosis the lipid accumulation appears to spread into the intimae adjacent to plaque as well as into the midiae below the plaques involving elastin in the elastic membranes of these $\operatorname{areas}^{9,10}$. As elastin is the primary site within the vascular wall for degenerative dis- 
orders, it is often hypothesized that changes in elastin's biochemical environment, such as exposure to various lipids, may be an important contributor to arterial stiffening ${ }^{11,12}$.

Elastin consists of hydrophobic regions that are rich in glycine, proline, and valine as well as cross-linking domains, comprised of desmosine and isodesmosine, that are often surrounded by polyalanine ${ }^{13,14}$. To understand the mechanism of elasticity of this protein, several models have been proposed based on short repeating motifs. In recent years, the repeating motif $(V P G V G)_{n}$, which repeats $n=9$ times in human elastin ${ }^{15}$, has received a great deal of attention. Luan et al. proposed the librational entropy mechanism (LEM) using both experimental and theoretical studies on the polypentapeptide VPGVG. According to this model the motif VPGVG is characterized by type II $\beta$-turns whose amplitude of libration is reduced upon extension. The changes in the amplitude of libration give rise to a decrease in entropy resulting in an elastomeric force ${ }^{16}$. Hong and coworkers performed a number of studies on this elastic mimetic polypetide. In one of their studies, proline and glycine isotropic and anisotropic chemical shifts indicated the presence of type II $\beta$ turns ${ }^{17}$. In a following study, they reported the dynamics of an elastin-mimetic protein, $\left[(\mathrm{VPGVG})_{4}(\mathrm{VPGKG})\right]_{39}$, as a function of hydration by solid state NMR spectroscopy. It was shown that water induces segmental motion and that the polypeptide exhibits large amplitude dynamical characteristics at and above $30 \%$ hydration levels ${ }^{18}$.

Environmental factors such as the degree of hydration, polarity of the solvent, and temperature are known to affect the dynamical characteristic of this remarkable protein. ${ }^{13} \mathrm{C}$ NMR studies of calf ligamentum nuchae, by Torchia and coworkers, showed that elastin consists of highly mobile chains ${ }^{19}$. A following study supported this finding with relaxation data which indicated that there is high mobility in this protein while the molecular motion is restricted in the alanine-rich regions ${ }^{20}$. Gotte and coworkers proposed that elastin may behave like rubber under certain conditions; however, this property can be lost upon complete dehydration. Kumashiro et al. observed the ${ }^{13} \mathrm{C}$ NMR spectra in elastin with different hydration levels at various temperatures. Samples with high hydration levels were observed 
to exhibit highly mobile characteristics; however, rigid solid-like characteristics are observed at low hydration levels and at temperatures below the freezing point of water ${ }^{21}$. Recent studies of the effects of a solvent such as glycerol by Demuth et al. have demonstrated that the protein mobility may be governed by solvent viscosity and showed evidence for a strong coupling between protein fluctuations and solvent dynamics ${ }^{22}$.

Lillie and Gosline showed that biochemical solvents have an important effect on the elastic and viscoelastic behavior of elastin. An increased tensile storage and loss modulus was observed and the tissue became stiffer when treated with glucose ${ }^{23}$. In another study, they showed that the hydration of the elastin network decreased at low sodium dodecyl sulfate (SDS) levels and increased at higher SDS levels ${ }^{4}$. Our recent work probed microscopic dynamical and structural modifications of porcine aortic elastin exposed to glucose, using solid-state spectroscopic and relaxation methodologies. Additionally, macroscopic stress strain tests were performed and revealed that glucose treated samples were mechanically stiffer than the same tissue without glucose treatment. Results from ${ }^{13} \mathrm{C}$ magic-angle spinning NMR methods indicated that glucose exposure gives rise to reduced mobility throughout the protein. Additionally, two dimensional ${ }^{2} \mathrm{H} T_{1}-T_{2}$ experiments indicated that the tumbling motion of water in these samples were similar; however, differences were observed between the relative populations of water across the samples. Simulations were also performed to probe the effects of glucose on elastin and showed that stiffening arises from an energetic contribution to the retractive force in the hydrophobic VPGVG domains of the protein ${ }^{24}$.

In this work we report on the changes in macroscopic properties of elastin following exposure to cholesterol. Elastin may exhibit a decrease in stiffness following cholesterol treatment, and dimensional changes are observed which suggest micro-structural alterations. We study the dynamical and structural modifications of cholesterol treated porcine aortic elastin compared with untreated samples, using ${ }^{13} \mathrm{C}$ magic angle spinning spectroscopy and relaxation NMR methodologies. In addition, the dynamics and populations of water in untreated as well as cholesterol treated samples were investigated using $2 \mathrm{D}{ }^{2} \mathrm{H} T_{1}-T_{2}$ 
NMR techniques. Lastly, we present results from molecular dynamics simulations on the repeating motif VPGVG in cholesterol solution and in water to give insight into structural and dynamical alterations which may contribute to changes in the proteins' elastomeric characteristics.

\section{Materials and Methods}

\section{Sample Preparation}

Fresh porcine thoracic aorta was collected from a local abattoir. Porcine aorta was chosen because of its availability and anatomical similarity to humans ${ }^{25}$. The sample was prepared within $24 \mathrm{~h}$ of acquisition to minimize the natural deterioration of the artery and its constituents. The artery was cleaned of blood, fat, and adherent tissues. The cleaned artery was then carefully cut open along the longitudinal direction and then into square pieces with dimensions of about $20 \mathrm{~mm} \times 20 \mathrm{~mm}$. Samples were then washed with distilled water. Elastin was isolated from the tissue by cyanogen bromide $(\mathrm{CNBr})$ treatment ${ }^{26}$. Arterial samples were submerged in $50 \mathrm{mg} / \mathrm{mL} \mathrm{CNBr}$ solution in $70 \%$ formic acid. For every square $\mathrm{cm}$ of tissue $8 \mathrm{~mL}$ of solution was used. After $19 \mathrm{~h}$ of treatment at room temperature and constant stirring, the samples were stirred in the same solution for $1 \mathrm{~h}$ at $60^{\circ} \mathrm{C}$, followed by 5 minutes of boiling to inactivate $\mathrm{CNBr}$. The purified samples were transferred to $1 \times$ phosphate buffered saline (PBS) solution $\left(137 \mathrm{mM} \mathrm{NaCl}, 2.7 \mathrm{mM} \mathrm{KCl}, 4.3 \mathrm{mM} \mathrm{Na} 2 \mathrm{HPO}_{4}, 1.47 \mathrm{mM}\right.$ $\mathrm{KH}_{2} \mathrm{PO}_{4}$ ) prior to experiments. Throughout this work, purified elastin in $1 \times \mathrm{PBS}$ solution is

referred to as the 'untreated' sample and that treated with cholesterol (as described above) is denoted as the 'cholesterol treated' sample.

\section{Preparation of cholesterol treated elastin}

A stock solution of cholesterol (Matreya LLC) $(100 \mu \mathrm{g} / \mathrm{mL})$ in $1 \%$ taurodeoxycholic acid, sodium salt (Santa Cruz Biotechnology) was prepared by following the procedure developed 
by Hornebeck and Partridge ${ }^{27}$. For cholesterol treatment of elastin, taurodeoxycholic acid, sodium salt was used to dissolve cholesterol in order to form an optically clear solution of $1 \%$ taurodeoxycholic acid solution because cholesterol is insoluble in water. $100 \mathrm{~mL}$ of $1 \%$ aqueous taurodeoxycholic acid, sodium salt solution was mixed with $1 \%$ solution of cholesterol dissolved in ethanol $(1 \mathrm{~mL})$ under constant stirring for $3-4$ days. The isolated elastin samples were placed in beakers filled with the prepared stock solutions. The treated samples were incubated under constant magnetic stirring at $37^{\circ} \mathrm{C}$ for 7 days. The sample side lengths and thickness were measured before and after the incubation. All measurements were made using a digital caliper.

\section{Mechanical testing}

The mechanical behavior of tissue samples before and after cholesterol treatment was measured using a biaxial tensile tester, which has been utilized in previous studies of soft tissue mechanics ${ }^{2,28,29}$. To load the sample onto the biaxial tensile tester, each side was clamped into sandpaper-lined grips. Then the samples were hooked on all four sides and attached with nylon sutures. Four black carbon beads were glued as marker dots onto the surface of the elastin samples in the shape taking the space of about $10 \mathrm{~mm} \times 10 \mathrm{~mm}$ square. The CCD camera mounted on the biaxial tensile tester was used to track the markers for measurement of both circumferential and longitudinal stretching, as loads were applied onto the samples. The sutures on the samples were connected to carriages with load cells mounted in orthogonal directions to allow users to control loading using LabVIEW. During the tensile tests untreated and treated tissue samples were submerged in PBS and cholesterol solution, respectively. Elastin samples were preconditioned by 15 seconds duration loading and unloading cycles, repeated eight times, by applying a tension of $40 \mathrm{~N} / \mathrm{m}$ equibiaxially for repeatability of the experiment ${ }^{2}$. Once the testing specimen was preconditioned, a minimal preload $5.00 \pm 0.05 \mathrm{~N} / \mathrm{m}$ was applied to maintain the suture's tautness. Once the preload was applied, equibiaxial loads of $100 \mathrm{~N} / \mathrm{m}$ were applied for 15 second duration loading and 
unloading stages, repeated eight times, equi-biaxially on the elastin samples. Cauchy stress and stretch were determined in the longitudinal and circumferential directions to characterize the elastic behavior of elastin ${ }^{30}$.

\section{Statistical analysis}

In order to compare the dimensional and tangent modulus changes with progression of cholesterol deposition, the measured dimensions and tangent modulus of elastin after incubation were normalized with respect to the initial dimensions and tangent modulus measured before the incubation. Normalized dimensional changes, tangent modulus, and stress-stretch data are plotted with mean \pm standard deviation. Statistical analysis was performed by JMP Pro (version 10.0.2, SAS Institute Inc.) using a two-tail two sample t-test (assuming unequal variances, differences are considered significant when $p<0.05)$.

\section{${ }^{13} \mathrm{C}$ NMR relaxation and spectroscopy}

${ }^{13} \mathrm{C}$ cross-polarization (CP) and direct-polarization (DP) experiments were performed on a 17.61 Tesla Bruker Avance system (Billerica, MA) at $37^{\circ} \mathrm{C}$ using a $3.2 \mathrm{~mm}$ rotor and $10 \mathrm{kHz}$ magic angle spinning (MAS). The rotors were sealed using compression-style rotor caps in all the studies. Sample temperature was regulated to within $1^{\circ} \mathrm{C}$ and the rotor spinning speed was regulated to within $5 \mathrm{~Hz}$. For the $\mathrm{CP}$ experiments, a $2.5 \mathrm{~ms}$ ramp on the ${ }^{1} \mathrm{H}$ channel was used. All ${ }^{13} \mathrm{C}$ experimental data were acquired with $8 \times 10^{4} \mathrm{~Hz}$ of ${ }^{1} \mathrm{H}$ TPPM decoupling ${ }^{31}$. The acquisition time in all studies was $15 \mathrm{~ms}$ and the spectral width was set to $299.5 \mathrm{ppm}$. All data were processed using $25 \mathrm{~Hz}$ of Gaussian broadening and the ${ }^{13} \mathrm{C}$ chemical shifts were referenced to adamantane (tetramethylsilane scale). For the ${ }^{13} \mathrm{C} T_{1 \rho}$ relaxation experiments, spin-locking fields of $30 \mathrm{kHz}$ and $59 \mathrm{kHz}$ were used for the $\mathrm{CP}$ experiments on the untreated samples. Spin locking fields of $32 \mathrm{kHz}$ and $67 \mathrm{kHz}$ were used for the $\mathrm{CP}$ experiments for the cholesterol treated sample. All ${ }^{13} \mathrm{C} T_{1 \rho}$ experiments made use of pulse durations ranging from $50 \mathrm{~ms}$ to $8.5 \mathrm{~ms}$. In these relaxation measurements, the ${ }^{13} \mathrm{C}$ carrier during the locking pulse 
was offset to either $35 \mathrm{ppm}$ or $173 \mathrm{ppm}$ for measuring the aliphatic or carbonyl relaxation times, respectively. In water hydrated elastin, ${ }^{13} \mathrm{C} T_{1}$ relaxation times are on the order of 1 $\mathrm{s}^{31,32}$. Thus, all measurements in this study were performed with a recycle time of $5 \mathrm{~s}$.

\section{${ }^{2} \mathrm{H}$ NMR relaxation}

The ${ }^{2} \mathrm{H} T_{1}-T_{2}$ experiments were run on a $200 \mathrm{MHz}$ Varian Unity system (Palo Alto, CA) at $37^{\circ} \mathrm{C}$. The same samples used in the ${ }^{13} \mathrm{C}$ NMR studies were soaked in ${ }^{2} \mathrm{H}_{2} \mathrm{O}$ for a duration of $24 \mathrm{~h}$ before experiments were run. In these two-dimensional relaxation measurements, the interpulse spacing in the CPMG train was $700 \mu \mathrm{s}$ and the ${ }^{2} \mathrm{H} \pi / 2$ pulse was $28 \mu$ s. Stroboscopic detection of 6000 echo peaks was used to measure the $T_{2}$ relaxation time, and 100 logarithmically spaced points ranging from $1 \mathrm{~ms}$ to $10 \mathrm{~s}$ were sampled in the $T_{1}$ dimension. To analyze the ${ }^{2} \mathrm{H} T_{1}-T_{2}$ relaxation data, we applied a two-dimensional inverse Laplace transform (ILT) algorithm described elsewhere ${ }^{33}$.

\section{Simulations}

Molecular dynamics simulations were performed in GROMACS ${ }^{34}$ using the GROMOS 53A6 force field ${ }^{35}$ and the SPC-216 water model ${ }^{36}$. The peptide $[\mathrm{VPGVG}]_{5}$ was simulated at a temperature of $310 \mathrm{~K}$ in water, and in a $1 \%$ by volume cholesterol solution to match the experimental conditions in this study. A stretching force was added to additional simulations in order to determine retractive forces. The peptides were terminated as neutral molecules, and the velocity-rescaling thermostat ${ }^{37}$ was used for the simulations. The simulations ran for an equilibration time period of $20 \mathrm{~ns}$, with the final frame being used as a starting structure for the stretched and unstretched simulations. For the stretched studies, the peptides were first stretched for $2 \mathrm{~ns}$ using an umbrella potential and a rate constant of $5000 \mathrm{~kJ} / \mathrm{mol} / \mathrm{nm}^{2}$. After this step, the peptides were held for 4 ns with a constant force potential using a rate constant of $1000 \mathrm{~kJ} / \mathrm{mol} / \mathrm{nm}^{2}$. The unstretched simulations were performed for an additional 60 ns and these additional times were used for all calculations. 


\section{Results and Discussion}

\section{Mechanical studies of cholesterol treated elastin}

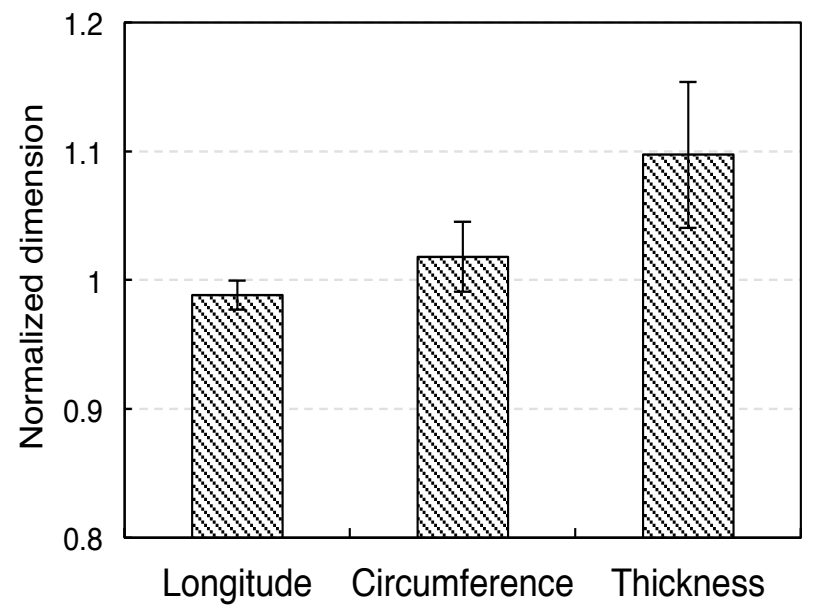

Figure 1: Longitude, circumference, and thickness of elastin treated with cholesterol for 7 days $(n=6)$. The sizes of elastin samples after treatment were normalized to the sizes before treatment. ( $p=0.7228,0.3060$, and 0.1092 for the longitudinal, circumferential, and thickness directions, respectively).

The effects of cholesterol on the mechanical properties of elastin were studied by equibiaxial tensile tests. As shown in Figure 1, cholesterol treatment results in a slightly increased thickness after 7 days of treatment. The dimensional changes, however, are small and may be obscured by the variance among the samples. The overall swelling effect of cholesterol treatment indicates an alteration in the microstructure of elastin. Figure 2 shows a comparison of Cauchy stress versus stretch curves of elastin before and after treatment. For quantitative comparison of the effects of changes in solution composition on elastin's stiffness, the tangent modulus was obtained by differentiating the stress-stretch curves. The normalized tangent modulus, as shown in Figure 3, was obtained by dividing the tangent modulus after treatment by the modulus before treatment. Changes in the stress-stretch curves are not statistically significant, but show a clear trend in the cholesterol treated sample behaving with less stiffness than the untreated sample. Because ethanol was used to dilute the taurodeoxycholic acid, additional mechanical measurements were performed in a 
A)

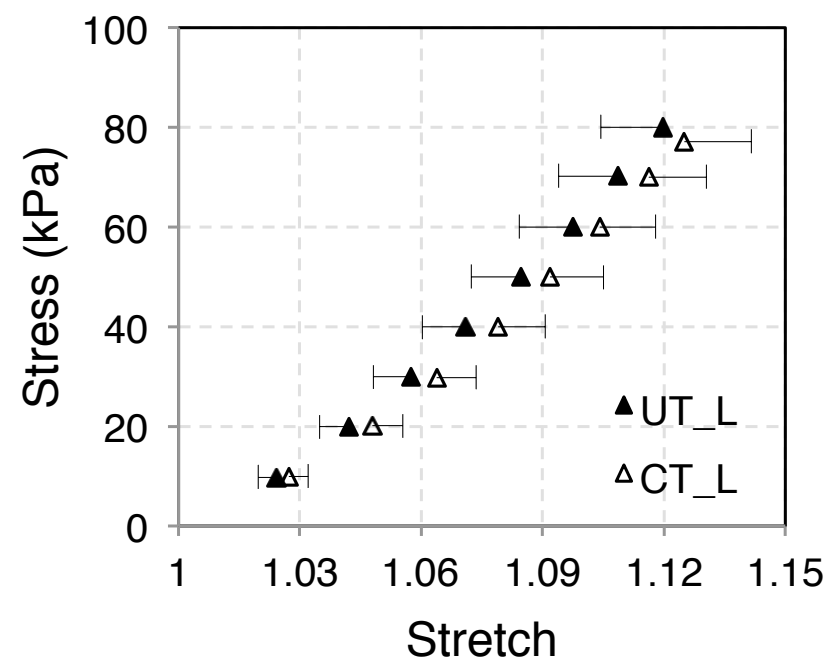

B)

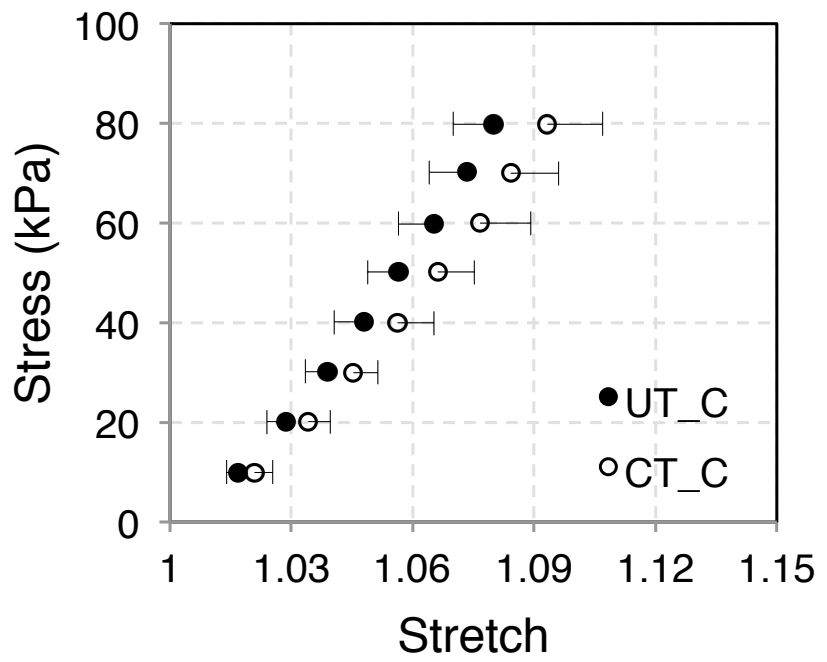

Figure 2: Average Cauchy stress versus stretch curves of untreated (UT) and cholesterol treated $(\mathrm{CT})$ elastin along the A) longitudinal $(\mathrm{L}, p=0.7159)$ and $\mathrm{B})$ circumferential $(\mathrm{C}$, $p=0.4735)(\mathrm{n}=6)$.

water-ethanol mixture (at the same concentration) without cholesterol to verify that changes due to ethanol exposure were negligible. These measurements (data not shown) show that the water-ethanol solution has a very slight effect on the mechanical properties of elastin. Therefore, the minute amount of ethanol used in preparation of cholesterol is not responsible for the reduced stiffness, and the changes observed arise from the effect of cholesterol treatment. Additional mechanical measurements were performed on the control sample in PBS at 14 and 28 days (data not shown) showed negligible changes in the stress-strain responses.

\section{${ }^{13} \mathrm{C}$ NMR of cholesterol treated elastin}

Table 1 introduces the ${ }^{13} \mathrm{C}$ chemical shifts and related secondary structures from different studies in the literature (Reprinted from reference ${ }^{24}$, with permission from Elsevier). These data were taken from atomic coordinates in the Protein Data Bank and ${ }^{13} \mathrm{C}$ NMR chemical shift data reported for 40 proteins, a protocol based on the joint probability of secondary structures tested on 36 proteins, ${ }^{13} \mathrm{C}$ labeled Nephila clavipes dragline silk, and 


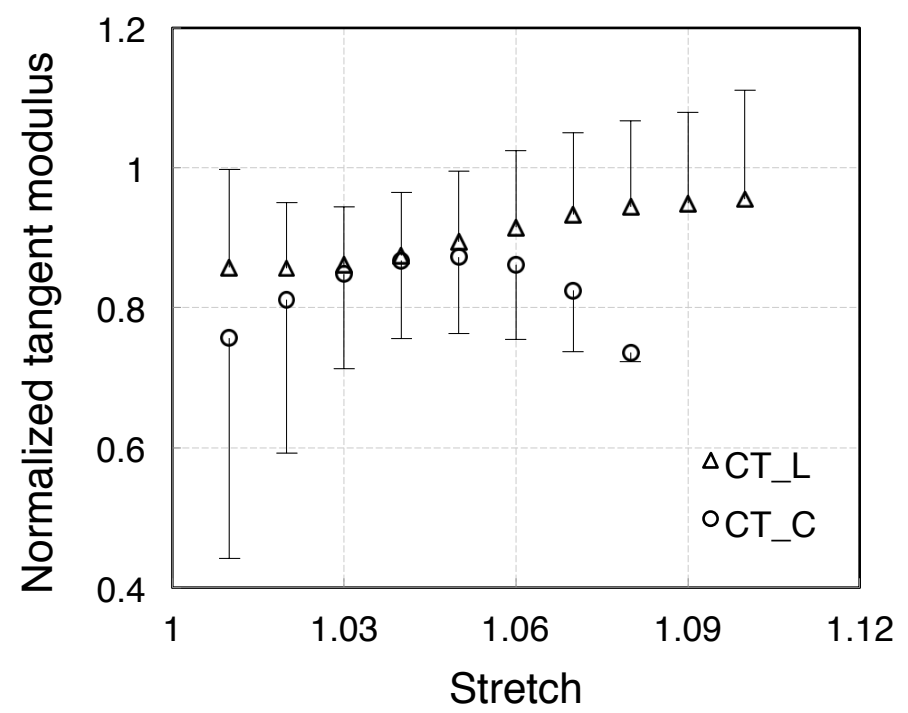

Figure 3: Normalized tangent modulus of cholesterol treated (CT) elastin in the longitudinal $(\mathrm{L}, p=0.3038)$ and circumferential $(\mathrm{C}, p=0.1404)$ directions $(\mathrm{n}=6)$.

a secondary structure database of reference corrected protein chemical shifts derived from the BioMagResBank. The ${ }^{13} \mathrm{C}$ chemical shift data for the $\mathrm{C}_{\delta}$-isoleucine and $\mathrm{C}_{\gamma}$-valine sites were obtained from an experimental study on a protected linear hexapeptide [Gly-Gly-XY-Gly-Gly]. Table 2 highlights the spectral assignments from our study based on Table 1.

Tropoelastin is a large $72 \mathrm{kDa}$ protein that is cross-linked together by desmosine and isodesmososine to form the essentially infinite macromolecule we refer to as elastin. The size of elastin together with its insoluble nature makes experimental studies (e.g. by present day NMR methods) of its structure challenging. Neverthelesss, several studies have reported on ${ }^{13} \mathrm{C}$ MAS NMR methods to study the structure of elastin and its associated peptides. In this work we summarize differences between the cholesterol and control samples, and refer the reader to the detailed chemical shift assignments and discussion of observable structures elsewhere $^{38,39}$. The most abundant amino acids of elastin are proline (11.4\%), valine (13.5\%), alanine $(22.9 \%)$, and glycine $(31.4 \%)$. According to a two-dimensional double quantum spectrum of Poly(Lys-25) $\left[(\mathrm{VPGVG})_{4}(\mathrm{VPGKG})\right]_{39}$, Yao et al. suggest that the peak at 19 ppm points to $C_{\gamma}$-valine sites ${ }^{18}$. For bovine neck ligament elastin, however, Pometun et al. 
assigned the peak at $19 \mathrm{ppm}$ to the $C_{\beta}$-alanine sites. Further, in that work, they reported that the peaks at 31.83 and $32.80 \mathrm{ppm}$ arise from side chains of proline and valine. The $C_{\alpha^{-}}$ proline and $C_{\alpha^{-}}$-valine were observed at 63.35 and $62.08 \mathrm{ppm}$, respectively ${ }^{39}$. According to Kumashiro et al. the $C_{\alpha}$-alanine signal at $53 \mathrm{ppm}$ points to an $\alpha$ helical structure ${ }^{40}$. Based on the tabulated chemical shifts presented in Table 1, our previous studies have shown that the $C_{\alpha}$-alanine peak at $53 \mathrm{ppm}$ may be indicative of either $\alpha$-helical or random coil structure ${ }^{24,38}$.

Figure 4 shows the aliphatic and carbonyl regions of the ${ }^{13} \mathrm{C}$ direct polarization spectra for the untreated and cholesterol treated samples. There is an overall reduction in resolution throughout the entire aliphatic spectrum in the cholesterol treated sample. These changes may result from an increase in the structural heterogeneity of this large, highly cross-linked protein, or from enhanced dynamics on the magic angle spinning time scale. Changes in dynamics are likely to contribute less as the ${ }^{1} \mathrm{H}^{13} \mathrm{C}$ correlation times measured (discussed below) are on the order of $\mu$ s and vary by factors of only 2-3 between the untreated and cholesterol treated sample. The spectral broadening in the cholesterol treated sample in some cases obscures features which are well resolved in the untreated sample. For example, peaks assigned to the $\alpha$-carbon of valine and proline (at approximately $60 \mathrm{ppm}$ ), as well as the $\mathrm{C}_{\beta}$-phenelyalanine, $\mathrm{C}_{\beta}$-isoleucine peaks at $41 \mathrm{ppm}$ are obscured. The large peak at $43 \mathrm{ppm}$, assigned to the $\alpha$-carbon of glycine, the most abundant amino acid of elastin, appears in the same position for both samples, but is broader in the cholesterol treated sample. This chemical shift does not point to a well defined secondary structure and may arise from $\alpha$-helical, $\beta$-spiral, and/or random coil structures. $\mathrm{C}_{\beta}$-proline and $\mathrm{C}_{\beta}$-valine peaks at approximately $31 \mathrm{ppm}$ appear broader in the cholesterol treated sample, but appear to have the same chemical shift. The highest peak observed at 18-20 ppm in the aliphatic region in both samples arises from the $\mathrm{C}_{\beta}$-alanine moieties; no significant chemical shift changes greater than $0.5 \mathrm{ppm}$ were observed between the samples for this peak. One notable difference in these spectra is the appearance of an additional signal observed at $13.6 \mathrm{ppm}$ in the cholesterol treated sample which was not observed in the untreated sample. This peak 
extends beyond the values expected for $\mathrm{C}_{\delta}$-isoleucine chemical shifts, but may arise from a methyl carbon signal from taurodeoxycholic acid. In previous studies a methyl carbon chemical shift of $15.42 \mathrm{ppm}$ was measured for taurodeoxycholic acid in ${ }^{2} \mathrm{H}_{2} \mathrm{O}^{41}$.

Figure 5 shows the ${ }^{1} \mathrm{H}-{ }^{13} \mathrm{C}$ cross polarization spectra of untreated and cholesterol treated samples. A well documented characteristic of hydrated elastin at physiological temperature is its high mobility. This mobility, in part, is believed to allow for configurational entropy changes which give rise to its elastomeric properties ${ }^{42,43}$. In a hydrated state and physiological temperature, the highly mobile nature of the protein backbone has been observed ${ }^{44,45}$ to lead to a small yet observable ${ }^{1} \mathrm{H}_{-}{ }^{13} \mathrm{C}$ cross-polarization signal (in a rigid solid the theoretical enhancement factor is approximately $4^{46}$ ). Here we compare the efficiency of the cross-polarized signal to probe dynamical changes across the samples; in the case of isotropic fast motion the ${ }^{1} \mathrm{H}-{ }^{13} \mathrm{C}$ dipolar interactions would be averaged and no cross-polarized signal would be expected. These measurements reveal that the cholesterol treated sample cross polarizes slightly less in comparison with the untreated sample pointing to an increase in dynamical characteristics. Peaks that appear in the untreated sample at approximately 61.9 $\operatorname{ppm}\left(\mathrm{C}_{\alpha}\right.$-proline and $\mathrm{C}_{\alpha}$-valine $), 50.9 \mathrm{ppm}$ ( $\mathrm{C}_{\alpha}$-alanine $), 43-44 \mathrm{ppm}$ ( $\mathrm{C}_{\alpha}$-glycine $)$, and 31 $\operatorname{ppm}\left(\mathrm{C}_{\beta}\right.$-proline and $\mathrm{C}_{\beta}$-valine $)$ are not observable in the spectra of the cholesterol treated sample. In our recent work, we reported dynamical and structural changes of elastin after glucose exposure by ${ }^{13} \mathrm{C}$ magic angle spinning NMR methodology. The experimental results indicated that glucose treated elastin cross polarizes more efficiently than the untreated samples, pointing to more rigid structures; however, we observed the reverse effect following cholesterol treatment. In contrast to the present study, glucose exposure led to an important chemical shift differences-at $53.4 \mathrm{ppm}\left(\mathrm{C}_{\alpha}\right.$-alanine), pointing to an increased $\alpha$ helical character in the alanine motifs ${ }^{24}$. 
Table 1: Ranges of the $\mathrm{C}_{\alpha}, \mathrm{C}_{\beta}$, and $\mathrm{C}_{\gamma}$ chemical shifts for the most abundant amino acids in elastin. Shown are the maximum and minimum chemical shifts observed in the literature ${ }^{47-52}$. In the table, the maximum value was determined from the largest reported value plus its uncertainty, and for the minimum the smallest reported value minus its uncertainty. All values except ${ }^{47}$ were adjusted for the TMS scale by subtracting $1.7 \mathrm{ppm}^{52}$ as the original references used DSS as the standard. Values indicated with a $\dagger$ are provided when the amino acid is followed by alanine and $\ddagger$ are evaluated when followed by proline. Reprinted from reference ${ }^{24}$, with permission from Elsevier.

\begin{tabular}{|c|c|c|c|}
\hline Assignment & $\alpha$-helix $[\mathrm{ppm}]$ & $\beta$-sheet/turn $[\mathrm{ppm}]$ & random coil $[\mathrm{ppm}]$ \\
\hline$C_{\delta}$-Ile & - & - & $11.2^{\dagger}$ \\
\hline$C_{\gamma}$-Val & - & - & $18.4-19.2 \ddagger$ \\
\hline$C_{\beta}$-Ala & $14.6-17.65$ & $17.39-21.49$ & $16.06-18.62$ \\
\hline$C_{\beta}$-Ile & $34.75-37.05$ & $36.18-40.24$ & $34.50-38.64$ \\
\hline$C_{\beta}$-Leu & $38.59-41.00$ & $40.09-44.31$ & $38.47-42.32$ \\
\hline$C_{\beta}$-Phe & $35.72-38.70$ & $38.10-41.59$ & $35.77-39.73$ \\
\hline$C_{\beta}$-Pro & $28.54-30.71$ & $29.37-31.77$ & $29.21-31.19$ \\
\hline$C_{\beta}$-Thr & $65.96-68.35$ & $67.01-71.23$ & $66.76-69.75$ \\
\hline$C_{\beta}$-Val & $28.97-30.51$ & $30.32-33.90$ & $29.22-32.74$ \\
\hline $\mathrm{C}_{\alpha}$-Ala & $49.00-54.18$ & $47.90-51.31$ & $49.21-52.78$ \\
\hline $\mathrm{C}_{\alpha}$-Gly & $42.50-46.31$ & $41.50-44.69$ & $42.20-44.86$ \\
\hline $\mathrm{C}_{\alpha}$-Leu & $54.59-57.05$ & $51.05-53.69$ & $51.36-54.94$ \\
\hline $\mathrm{C}_{\alpha}$-Phe & $57.21-61.01$ & $53.32-56.54$ & $53.26-58.30$ \\
\hline $\mathrm{C}_{\alpha}$-Pro & $62.71-64.87$ & $59.87-62.31$ & $60.51-63.09$ \\
\hline $\mathrm{C}_{\alpha}$-Val & $62.87-66.01$ & $57.43-60.77$ & $57.85-62.52$ \\
\hline
\end{tabular}


Table 2: Measured ${ }^{13} \mathrm{C}$ chemical shifts and corresponding secondary structural assignments based on Table 1 for untreated and cholesterol treated porcine aortic elastin.

\begin{tabular}{|c|c|c|}
\hline ppm from TMS & Assigment & Structure \\
\hline \multicolumn{3}{|c|}{ Untreated Sample (Direct Polarization) } \\
\hline $11.9 \pm 0.5$ & $C_{\delta}$-Ile & r.c. \\
\hline $18.3 \pm 0.9$ & $C_{\beta}$-Ala, $C_{\gamma}$-Val & $\beta$-sheet/turn or r.c., r.c. \\
\hline $19.0 \pm 0.5$ & $C_{\beta}$-Ala, $C_{\gamma}$-Val & $\beta$-sheet/turn, r.c. \\
\hline $20.1 \pm 0.5$ & $C_{\beta}$-Ala, $C_{\gamma}$-Val & $\beta$-sheet/turn, r.c. \\
\hline $22.5 \pm 0.5$ & 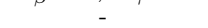 & - \\
\hline $24.0 \pm 0.5$ & - & - \\
\hline $25.7 \pm 0.5$ & - & - \\
\hline $31.4 \pm 0.9$ & $C_{\beta}$-Val, $C_{\beta}$-Pro &,-- \\
\hline $37.8 \pm 0.9$ & $C_{\beta}$-Phe, $C_{\beta}$-Ile & - , $\beta$-sheet/turn or r.c. \\
\hline $41.0 \pm 0.5$ & $C_{\beta}$-Phe, $C_{\beta}$-Leu & $\beta$-sheet/turn, - \\
\hline $44.2 \pm 0.9$ & $C_{\alpha}-\mathrm{Gly}, C_{\beta}-\mathrm{Leu}$ &,$- \beta$-sheet/turn \\
\hline $49.2 \pm 0.9$ & $C_{\alpha}$-Ala & $\beta$-sheet/turn or r.c. \\
\hline $51.3 \pm 0.7$ & $C_{\alpha}-\mathrm{Ala}, C_{\alpha}-\mathrm{Leu}$ & $\beta$-sheet/turn or r.c.,$\beta$-sheet/turn \\
\hline $53.8 \pm 0.9$ & $C_{\alpha}$-Ala, $C_{\alpha}-\mathrm{Phe}$ & $\alpha$-helix or r.c., r.c. or $\beta$-sheet/turn \\
\hline 56.2 & $C_{\alpha}$-Phe, $C_{\alpha}$-Leu & $\beta$-sheet/turn or r.c., $\alpha$-helix \\
\hline $58.0 \pm 0.3$ & $C_{\alpha}$-Val, $C_{\alpha}-\mathrm{Phe}$ & $\beta$-sheet/turn or r.c., $\alpha$-helix \\
\hline $60.9 \pm 0.5$ & $C_{\alpha}$-Val, $C_{\alpha}$-Pro & r.c. or $\beta$-sheet $/$ turn, r.c. or $\beta$-sheet $/$ turn \\
\hline $61.9 \pm 0.5$ & $C_{\alpha}$-Val, $C_{\alpha}$-Pro & r.c. or $\beta$-sheet/turn, - \\
\hline $68.7 \pm 0.5$ & $C_{\beta}-\mathrm{Thr}$ & $\beta$-sheet/turn or r.c. \\
\hline $71.2 \pm 0.5$ & $C_{\beta}-\mathrm{Thr}$ & $\beta$-sheet/turn \\
\hline \multicolumn{3}{|c|}{ Cholesterol Treated Sample (Direct Polarization) } \\
\hline $11.5 \pm 0.5$ & $C_{\delta}$-Ile & r.c. \\
\hline $13.7 \pm 0.5$ & - & - \\
\hline $17.9 \pm 0.5$ & $C_{\beta}$-Ala & r.c. or $\alpha$-helix \\
\hline $19.0 \pm 0.4$ & $C_{\beta}$-Ala, $C_{\gamma}$-Val & $\beta$-sheet/turn, r.c. \\
\hline $19.7 \pm 0.4$ & $C_{\beta}$-Ala, $C_{\gamma}-\mathrm{Val}$ & $\beta$-sheet/turn, r.c. \\
\hline $22.2 \pm 0.4$ & - & - \\
\hline $23.6 \pm 0.5$ & - & - \\
\hline $25.7 \pm 0.7$ & - & - \\
\hline $31.2 \pm 0.9$ & $C_{\beta}$-Val, $C_{\beta}$-Pro &,-- \\
\hline $36.4 \pm 0.9$ & $C_{\beta}$-Phe, $C_{\beta}$-Ile & -, r.c. or $\alpha$-helix \\
\hline $39.9 \pm 0.9$ & $C_{\beta}$-Phe, $C_{\beta}$-Leu &,-- \\
\hline $40.6 \pm 1.4$ & $C_{\beta}$-Phe, $C_{\beta}$-Leu & $\beta$-sheet/turn, - \\
\hline 41.7 & $C_{\alpha}-\mathrm{Gly}, C_{\beta}-\mathrm{Leu}$ &,-- \\
\hline $43.8 \pm 0.9$ & $C_{\alpha}$-Gly & - , $\beta$-sheet/turn \\
\hline $47.4 \pm 0.4$ & - & - \\
\hline $48.8 \pm 0.4$ & $C_{\alpha}$-Ala & $\beta$-sheet/turn \\
\hline $50.9 \pm 0.9$ & $C_{\alpha}$-Ala & $\beta$-sheet/turn or r.c. \\
\hline 53.8 & $C_{\alpha}-\mathrm{Ala}, C_{\alpha}-\mathrm{Phe}$ & r.c. or $\alpha$-helix, $\beta$-sheet/turn or r.c. \\
\hline 57.0 & $C_{\alpha}$-Phe & r.c. or $\alpha$-helix \\
\hline $61.9 \pm 2.0$ & $C_{\alpha}$-Val, $C_{\alpha}$-Pro & $\beta$-sheet/turn or r.c., - \\
\hline
\end{tabular}



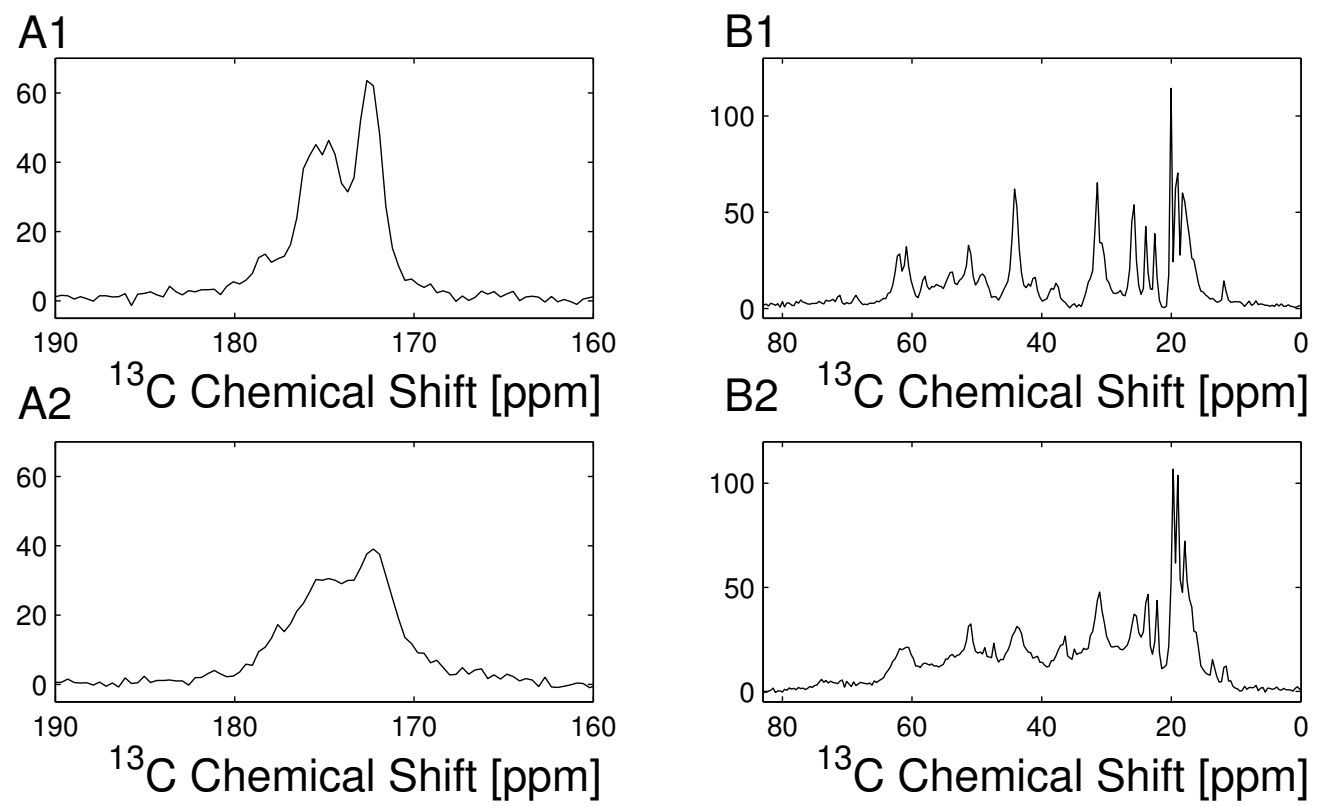

Figure 4: Direct polarization (DP) ${ }^{13} \mathrm{C}$ NMR spectra of untreated (A1-B1) and cholesterol treated (A2-B2) porcine aortic elastin samples acquired with decoupling at $10 \mathrm{kHz}$ magic angle spinning. Spectra labeled (B) correspond to the aliphatic region and spectra labeled (A) the carbonyl region. Differences are observed in the resolution of the various peaks between the samples, as discussed in the text. 

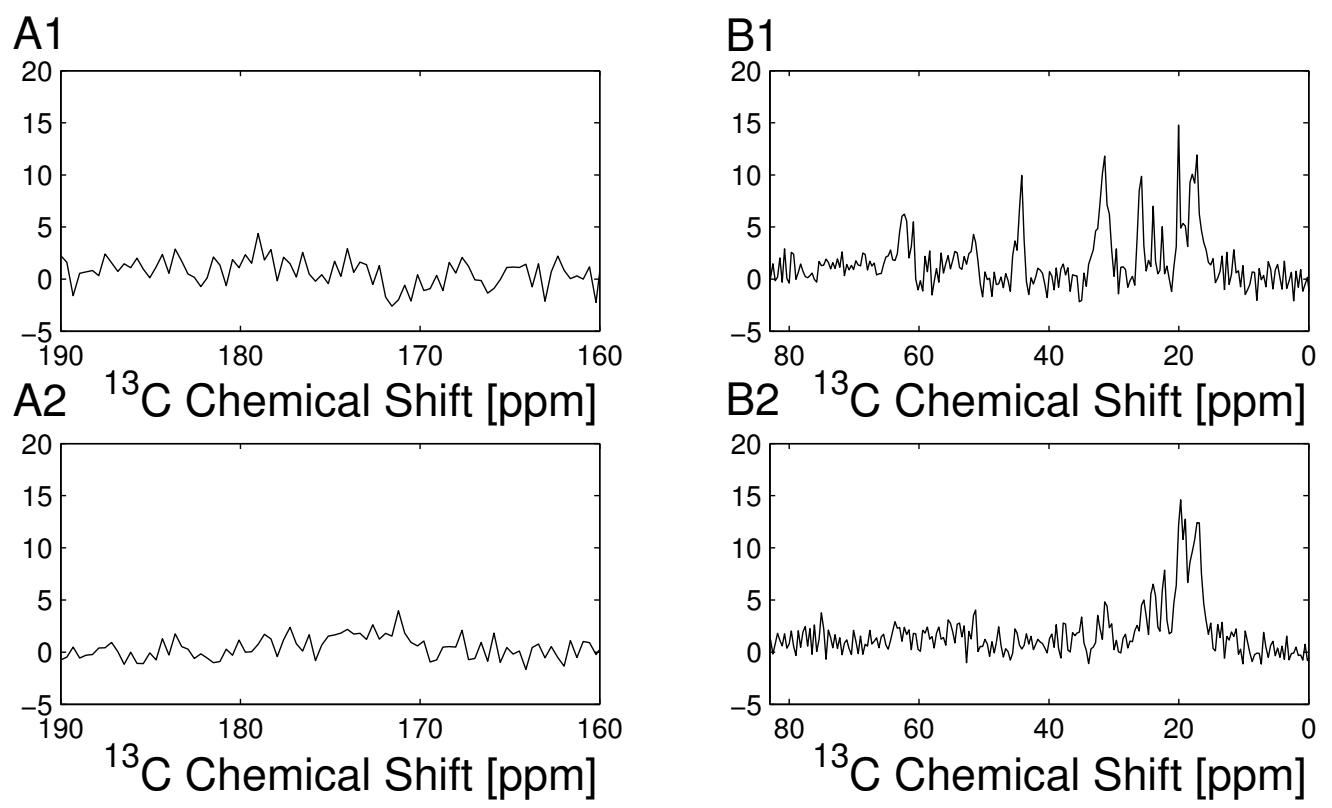

Figure 5: ${ }^{1} \mathrm{H}-{ }^{13} \mathrm{C}$ cross polarization $(\mathrm{CP}){ }^{13} \mathrm{C}$ NMR spectra of untreated (A1-B1) and cholesterol treated (A2-B2) porcine aortic elastin samples acquired with decoupling at 10 $\mathrm{kHz}$ magic angle spinning. 
To further quantify the dynamical characteristics of the cholesterol and untreated elastin samples we measured the ${ }^{13} \mathrm{C}$ NMR relaxation times in the rotating frame. Rotating frame relaxation times quantify dynamics over the time scale ranging from $\mu$ s to $\mathrm{ms}^{53}$. In these experiments, the ${ }^{13} \mathrm{C} R_{1 \rho}$ relaxation rate is given by ${ }^{54}$

$$
R_{1 \rho}^{I S}=R_{1}^{I S}+\sin ^{2} \theta_{\rho}\left(R_{1 \triangle}^{I S}-\frac{1}{2} R_{1}^{I S}\right)
$$

where $\theta_{\rho}$ is the angle between $B_{0}$ and $B_{1 e}$ (here $\theta_{\rho}=\pi / 2$ ). The $R_{1}^{I S}$ and $R_{1 \triangle}^{I S}$ are spin-lattice relaxation rates given by

$$
R_{1}^{I S}=\mu_{S}^{2}\left[J_{0}\left(\omega_{I}-\omega_{S}\right)+3 J_{1}\left(\omega_{I}\right)+6 J_{2}\left(\omega_{I}+\omega_{S}\right)\right]
$$

and

$$
R_{1 \triangle}^{I S}=\mu_{S}^{2}\left[3 J_{1}\left(\omega_{S}\right)+\frac{1}{3} J_{0}\left(\omega_{e}-2 \omega_{R}\right)+\frac{2}{3} J_{0}\left(\omega_{e}-\omega_{R}\right)+\frac{2}{3} J_{0}\left(\omega_{e}+\omega_{R}\right)+\frac{1}{3} J_{0}\left(\omega_{e}+2 \omega_{R}\right)\right] .
$$

The constant $\mu_{S}$ given by

$$
\mu_{S}=\hbar \gamma_{H} \gamma_{C} /\left(2 r^{3}\right)
$$

represents the dipolar coupling between proton and carbon nuclear spins. The spectral density is given by $J(\omega)=\left(1-x^{2}\right) \tau_{c} /\left(1+\omega^{2} \tau_{c}^{2}\right)$, where $x$ is a generalized order parameter that describes the amplitude of motion, and $\omega_{e}, \omega_{R}, \omega_{I}$, and $\omega_{S}$ represent the angular rotational frequency of the spin locking field, rotor, the proton, and the carbon Larmor frequencies, respectively. For this experiment we applied two different ${ }^{13} \mathrm{C}$ locking fields to determine the correlation time, $\tau_{c}$, that characterizes the motion of ${ }^{13} \mathrm{C}-{ }^{1} \mathrm{H}$ internuclear vectors. All relaxation measurements were performed with direct polarization, which gave a higher signal intensity for both untreated and cholesterol treated samples.

Table 3 highlights the relaxation times at two different locking fields and correlation times 
for the untreated and cholesterol treated samples. The $C_{\beta}$-valine, $C_{\beta}$-proline, $C_{\beta}$-alanine, and $C_{\gamma}$-valine correlation times appear smaller for the cholesterol treated sample than for the untreated sample, indicating faster motion for these moieties. Changes in the correlation times of the backbone carbonyl of the cholesterol treated sample are small, and are within our experimental uncertainty in comparison to the untreated sample.

We note that the model used in the determination of the correlation times tabulated in Table 3 assumes that fluctuations of ${ }^{13} \mathrm{C}^{-1} \mathrm{H}$ internuclear vectors, which were probed over the $\mu \mathrm{s}$ to ms time scale, may modeled by a single correlation time. Verifying the validity of this assumption would require measuring the spectral density with several relaxation measurements at different magnetic field strengths. Nevertheless, we checked the self-consistency of this assumption by taking the correlation time of the untreated sample and determined the $T_{1 \rho}$ relaxation time in the rotating frame at a different magnetic field measured by others for similar samples. Kumashiro and coworkers reported $T_{1 \rho}$ relaxation times for an elastin polypeptide from exons 20 and 23, interspersed with cross linking domains constructed from exons 21 and 23 (denoted EP20-24-24) ${ }^{40}$. Keeley and coworkers have shown that these short peptides may resemble various characteristics of elastin, including coacervation and elasticity ${ }^{55,56}$, and would therefore serve as a fair comparison to the experimental data in this work. Using the correlation times tabulated for the untreated sample, we computed the $T_{1 \rho}$ relaxation time at a magnetic field of approximately 9.4 Tesla (used in reference ${ }^{40}$ ). The measured $\mathrm{C}_{\alpha}$-glycine $T_{1 \rho}$ relaxation times in a Zeeman field of 9.4 Tesla was $2.0 \pm 0.1 \mathrm{~ms}$; using our measured $\mathrm{C}_{\alpha}$-glycine $T_{1 \rho}$ relaxation times and correlation time (see Table 3 ) we obtained $2.8 \mathrm{~ms}$, in reasonable agreement with the experimental value reported in ${ }^{40}$. As a second example, taking the measured correlation time and the model relaxation expressions, we find the the $C_{\beta}$-alanine, $C_{\gamma}$-valine relaxation times would be $16.3 \mathrm{~ms}$ at 9.4 Tesla in good agreement with the value reported $(12.9 \pm 1.1 \mathrm{~ms})^{40}$. We note that the model of a single correlation time failed to reproduce the measured spin-lattice relaxation times $\left(T_{1}\right)$, which probe dynamics over a longer time scale. This is due to the well-known characteristic of large 
proteins, such as elastin, exhibiting a diverse range of dynamics (e.g. acoustic modes ${ }^{43}$ ) and therefore require several correlation times to properly characterize the full spectral density.

Prior solid state NMR experimental studies of hydrated elastin have shown that that the line width of the carbonyl peak was approximately $3-4 \mathrm{kHz}$ in static samples, with ${ }^{1} \mathrm{H}$ decoupling but no magic angle spinning (see ${ }^{32,45}$ ). We note that this line width includes a contribution of the chemical shift anisotropy (CSA) as well as structural heterogeneity. It is worthwhile to inquire if the CSA is averaged at $10 \mathrm{kHz}$ magic angle spinning and how it may contribute to the ${ }^{13} \mathrm{C}$ relaxation process. Equations 1 through 4 assume only a ${ }^{1} \mathrm{H}_{-}{ }^{13} \mathrm{C}$ dipolar contribution to the relaxation process and appeared to provide reasonable agreement when performing a back calculation at a different Larmor field (above). Thus, the contribution of the CSA interaction appears to be negligible, further justifying the theoretical expressions used.

Table 3: Measured ${ }^{13} \mathrm{C} T_{1 \rho}$ relaxation times and correlation times determined for cholesterol treated and untreated elastin. The correlation times were determined from Equation 1, using a single correlation time to model the dynamical characteristics of ${ }^{1} \mathrm{H}_{-}{ }^{13} \mathrm{C}$ internuclear vectors.

\begin{tabular}{cccc}
\hline Assignment & $T_{1 \rho}[\mathrm{ms}]$ & $T_{1 \rho}[\mathrm{ms}]$ & $\tau_{c} \times 10^{-6}[\mathrm{~s}]$ \\
\hline Untreated Sample & $f_{e}=3.02 \times 10^{4}[\mathrm{~Hz}]$ & $f_{e}=5.87 \times 10^{4}[\mathrm{~Hz}]$ & \\
\hline$C_{\beta}$-Ala, $C_{\gamma}$-Val & $9.49 \pm 0.68$ & $20.04 \pm 1.10$ & $4.55 \pm 0.87$ \\
$22.5 \mathrm{ppm}$ & $11.57 \pm 0.84$ & $15.09 \pm 1.70$ & $1.93 \pm 0.91$ \\
$25.7 \mathrm{ppm}$ & $3.61 \pm 0.08$ & $4.70 \pm 0.17$ & $1.93 \pm 0.30$ \\
$C_{\beta}$-Val, $C_{\beta}$-Pro & $4.07 \pm 0.08$ & $5.37 \pm 0.18$ & $1.99 \pm 0.26$ \\
$C_{\beta}$-Phe, $C_{\beta}$-Ile & $2.37 \pm 0.08$ & $3.88 \pm 0.25$ & $3.08 \pm 0.54$ \\
$\mathrm{C}_{\alpha}$-Gly, $C_{\beta}$-Leu & $2.27 \pm 0.08$ & $3.16 \pm 0.23$ & $2.26 \pm 0.53$ \\
$\mathrm{C}_{\alpha}$-Ala, $\mathrm{C}_{\alpha}$-Leu & $2.07 \pm 0.04$ & $2.69 \pm 0.08$ & $1.92 \pm 0.25$ \\
$C=O$ & $1.01 \pm 0.02$ & $1.88 \pm 0.02$ & $3.52 \pm 0.28$ \\
\hline Cholesterol Treated Sample & $f_{e}=3.23 \times 10^{4}[\mathrm{~Hz}]$ & $f_{e}=6.66 \times 10^{4}[\mathrm{~Hz}]$ & \\
\hline 13.7 ppm & $2.55 \pm 0.23$ & $3.97 \pm 0.47$ & $2.35 \pm 0.89$ \\
$C_{\beta}$-Ala & $3.42 \pm 0.27$ & $6.98 \pm 1.03$ & $3.55 \pm 1.19$ \\
$C_{\beta}$-Ala, $C_{\gamma}$-Val & $2.85 \pm 0.45$ & $3.39 \pm 0.58$ & $1.25 \pm 0.70$ \\
$C_{\beta}$-Val, $C_{\beta}$-Pro & $0.92 \pm 0.09$ & $1.15 \pm 0.15$ & $1.46 \pm 0.94$ \\
$C_{\alpha}$-Gly, $C_{\beta}$-Leu & $0.88 \pm 0.08$ & $2.1 \pm 0.67$ & - \\
$C_{\alpha}$-Ala & $0.98 \pm 0.08$ & $1.58 \pm 0.21$ & \\
$C=O$ & $1.38 \pm 0.03$ & $2.57 \pm 0.06$ & $3.12 \pm 0.57$
\end{tabular}

\section{Water dynamics and distribution in cholesterol-treated elastin}

To study the dynamics and distribution of water in untreated and cholesterol treated samples, we used a $2 \mathrm{D} T_{1}-T_{2}$ NMR technique based on an inverse Laplace transform ${ }^{57}$. The $2 \mathrm{D} T_{1}$ $-T_{2}$ results are shown in Figure 6. Four reservoirs of water appear in the 2D map for each 
sample and are labeled $\alpha_{1}, \alpha_{2}, \beta$, and $\gamma$. Following our earlier work ${ }^{44}$, the peak $\alpha_{1}$ was assigned to bulk water which exhibits isotropic motion, as $T_{1} \approx T_{2}$. The peak denoted $\alpha_{2}$ corresponds to water likely between the elastic fibers; this was confirmed by blotting samples dry with a wipe which suppressed both peaks $\alpha_{1}$ and $\alpha_{2}$. Therefore, in what follows we focus our discussion on the population and dynamics of $\beta$ and $\gamma$ components. The ${ }^{2} \mathrm{H}_{-}{ }^{2} \mathrm{H}$ and ${ }^{1} \mathrm{H}-$ ${ }^{2} \mathrm{H}$ dipolar interactions are usually negligible in ${ }^{2} \mathrm{H}_{2} \mathrm{O}$ and the dominant relaxation pathway for ${ }^{2} \mathrm{H}$ nuclei is quadrupolar in origin (note that the static quadrupolar coupling constant of ${ }^{2} \mathrm{H}$ in ${ }^{2} \mathrm{H}_{2} \mathrm{O}$ is on the order of $200 \mathrm{kHz}$ ). Under these conditions, the $T_{1}$ and $T_{2}$ relaxation times are given by ${ }^{58}$

$$
\frac{1}{T_{1}}=\frac{3 \pi^{2}}{20} C_{Q_{\mathrm{eff}}}^{2}\left[2 J\left(\omega_{D}\right)+8 J\left(2 \omega_{D}\right)\right]
$$

and

$$
\frac{1}{T_{2}}=\frac{3 \pi^{2}}{20} C_{Q_{\mathrm{eff}}}^{2}\left[3 J(0)+5 J\left(\omega_{D}\right)+2 J\left(2 \omega_{D}\right)\right] .
$$

In the above equations, the constant $C_{Q_{\text {eff }}}$ is the effective quadrupolar coupling constant, given by

$$
C_{Q_{\mathrm{eff}}}=\frac{e^{2} Q_{\mathrm{eff}} Q}{h} \Gamma
$$

where $\Gamma$ is a motional averaging parameter. The spectral density is given by $J\left(\omega_{D}\right)=$ $\tau_{c} /\left(1+\left(\omega_{D} \tau_{c}\right)^{2}\right)$. The correlation time, $\tau_{c}$, quantifies the tumbling of the quadrupolar moment of the ${ }^{2} \mathrm{H}$ nucleus with the electric field gradient and is intramolecular in origin. Similar to the ${ }^{13} \mathrm{C}$ NMR relaxation measurements, the above model assumes the fluctuations (here, the relaxation times report on the interaction of the quadrupolar moment with the electric field gradient) measured on the NMR timescale are suitably modeled by a single correlation time. Verifying the validity of this assumption would require measurements at many different magnetic fields to measure the spectral density. Thus, we compare the measured correlation times within these assumptions. The correlation times were calculated using Equations 5 and 6, and are tabulated in Table 4. For the $\gamma$ component we find that correlation times 
are the same for the untreated $(11.35 \pm 2.00 \mathrm{~ns})$ and cholesterol $(11.00 \pm 2.85 \mathrm{~ns})$ treated samples. The measurements on the component $\beta$ may also indicate no difference between the untreated $(9.95 \pm 3.50 \mathrm{~ns})$ and cholesterol $(6.40 \pm 4.85 \mathrm{~ns})$ treated samples, within our experimental uncertainty.

Experiments have given insight into the mechanical behavior of elastin as well as to the contribution of a solvent in changing its elastomeric characteristics. Early work by Fogh et al. showed the relationship between mechanical work and heat exchange to explain the behavior of elastin according to the classical theory of rubber elasticity and hydrophobic interactions in elastin. They found that the presence of an organic solvent influences the thermodynamic and mechanical characteristics of the protein. Further, they suggested that the classical theory of rubber elasticity is not sufficient to explain the thermodynamic behavior of elastin, and proposed a model whereby elastic recoil arises from interactions between the hydrophobic groups in the protein and water at the interface ${ }^{59}$. Wittebort et al. applied ${ }^{13} \mathrm{C} \mathrm{NMR}$ methods to observe that the backbone of hydrated elastin exhibits large amplitude motions, which contribute to its elastomeric properties ${ }^{39}$. Lillie and Gosline used SDS to study the effect of lipid-induced changes on the viscoelastic behavior of elastin and showed that low SDS levels cause a decrease in the hydration of the elastin network, while higher SDS levels had the opposite effect. Additionally, elastin exhibits softer mechanical characteristics in a hydrated network but is stiff in a dehydrated network ${ }^{4}$. We measured the concentration of water using the integrated intensity in the two-dimensional ILT map shown in Figure 6 relative to the dry lyophilized sample weight (shown as arbitrary units per gram of sample $(\mathrm{AU} / \mathrm{g}))$. Note that the signal intensity shown in Figure 6 is on a logarithmic scale (see color key). The cholesterol treated sample was found to have a higher concentration of water than the untreated sample, and the change arises more so in the reservoir we have denoted by $\beta$. According to the correlation times tabulated in Table 4, water molecules in the reservoir denoted $\beta$ exhibit slightly smaller correlation times in comparison to water molecules in reservoir $\gamma$ and are thus in a less restricted environment. This two dimensional 
relaxation method does not allow us to identify or assign the observed water reservoirs to a particular structural domain of the protein. However, the changes observed appear to alter the populations and dynamics of the more mobile water molecules which are presumably less associated with any protein interactions (e.g. hydrogen bonding). Lastly, we note that the inverse Laplace algorithm that we used was tested in a prior work against simulation data (with five reservoirs) and varying the signal to noise ratio (see Figure 4 in $^{60}$ ). Briefly, we found that the algorithm did not accurately reproduce the expected $T_{1}$ and $T_{2}$ times and signal intensities when the signal to noise ratio was poor. In the present work we ensured that the signal intensity was adequate so that we didn't encounter this problem.

A)

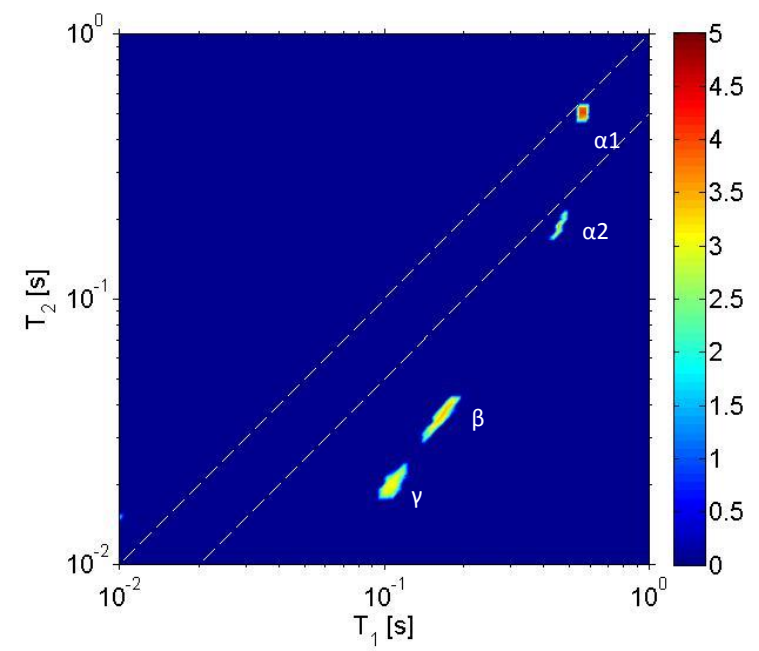

B)

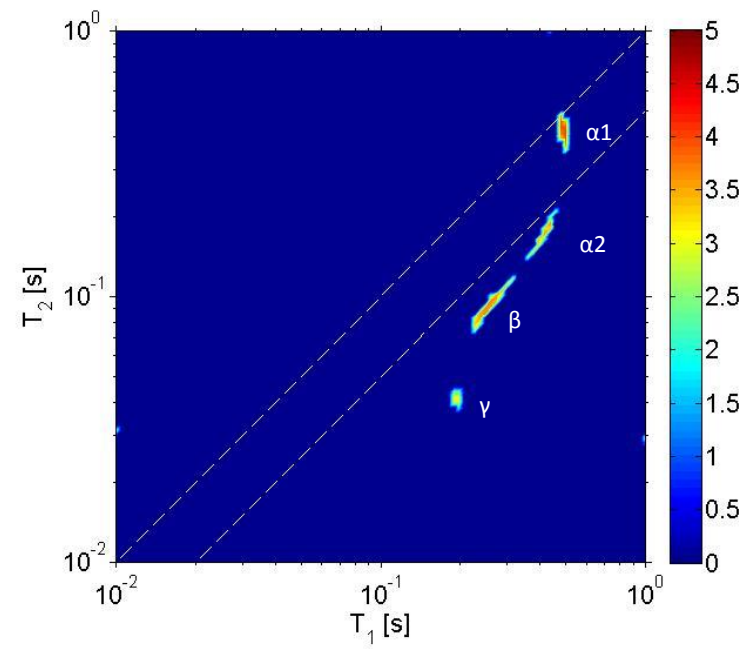

Figure 6: ${ }^{2} \mathrm{H} T_{1}-T_{2}$ results from untreated (A) and cholesterol-treated (B) porcine aortic elastin at $37^{\circ} \mathrm{C}$. Four distinguishable peaks are observed in each sample, denoted by $\alpha_{1}, \alpha_{2}$, $\beta$, and $\gamma$. Differences in the relative populations of water in the assigned $\gamma$ and $\beta$ regions are observed between the samples, as discussed in the text. The numerical values of the $T_{1}$ and $T_{2}$ times and the relative intensities are provided in Table 4. 
Table 4: Experimental ${ }^{2} \mathrm{H}$ relative signal intensities, relaxation times, and correlation times for the $\beta$ and $\gamma$ water components in cholesterol and untreated porcine aortic elastin at $37^{\circ} \mathrm{C}$. The number shown after the symbol \pm denotes half-width of the peak in the $T_{1}-T_{2}$ two dimensional map shown in Figure 6.

\begin{tabular}{ccc}
\hline & Untreated sample & Cholesterol treated sample \\
\hline$\gamma$ component & & \\
$T_{1}[\mathrm{~ms}]$ & $107.20 \pm 13.90$ & $191.85 \pm 13.85$ \\
$T_{2}[\mathrm{~ms}]$ & $20.44 \pm 2.20$ & $37.45 \pm 8.64$ \\
$\tau_{c}[\mathrm{~ns}]$ & $11.35 \pm 2.00$ & $11.00 \pm 2.85$ \\
$\beta$ component & & \\
$T_{1}[\mathrm{~ms}]$ & $163.00 \pm 33.30$ & $253.4 \pm 74.05$ \\
$T_{2}[\mathrm{~ms}]$ & $36.36 \pm 7.54$ & $92.18 \pm 30.22$ \\
$\tau_{c}[\mathrm{~ns}]$ & $9.95 \pm 3.50$ & $6.40 \pm 4.85$ \\
Signal intensity per unit dry sample mass $[\mathrm{AU} / \mathrm{g}]$ & & \\
$\gamma+\beta$ & 1.18 & 3.74 \\
$\gamma$ & 0.43 & 0.35 \\
$\beta$ & 0.75 & 3.39
\end{tabular}




\section{Molecular dynamics simulations of a short elastin repeat}

The total elastomeric restoring force $(f)$ of a material may be characterized by an energetic component $\left(f_{e}=\mathrm{d} U / \mathrm{d} r\right)$ and an entropic component $\left(f_{s}=-T \mathrm{~d} S / \mathrm{d} r\right), f=f_{s}+f_{e}{ }^{61}$. For an ideal elastomer, the energetic contribution is zero and $f=f_{s}$. For natural rubber and elastin, the ratios $f_{e} / f$ are 0.18 and 0.26 , respectively ${ }^{62}$. To understand the mechanism of elasticity, Luan et al. have proposed the librational entropy mechanism whereby the amplitude of the libration of a peptide chain is reduced upon extension, giving rise to a decrease in the entropy ${ }^{16}$. In this model, a harmonic oscillator of frequency $\omega_{i}$ is used to model atomic displacements yielding an entropy

$$
S_{i}=R\left[\ln \left(1-\mathrm{e}^{-\hbar \omega_{i} / k T}\right)^{-1}+\left(\hbar \omega_{i} / k T\right)\left(\mathrm{e}^{\hbar \omega_{i} / k T}-1\right)^{-1}\right] .
$$

A number of simulation studies of elastin peptides have been performed showing the entropic and energetic contributions to the retractive force. Wasserman and Salemme performed molecular dynamics simulations on $(\mathrm{VPGVG})_{18}$ in the relaxed and stretched states in water. The conformational entropy was found to reduce upon extension, and they suggested that hydrophobic interactions are the major component for elastic entropy at small extensions, whereas the librational mechanism contributes to the elastic restoring force at long extensions ${ }^{63}$. Chang and Urry showed via molecular dynamics simulations that changes in the energetic contribution to the retractive force $\left(f_{e}\right)$ of the elastin mimetic peptide VPGVG are small in comparison to changes in entropy $\left(f_{s}\right)^{42}$. We also recently reported experimental studies of elastin under strained and relaxed conditions. The experiments showed that under uniaxial strain the surrounding waters of hydration of elastin upon extension undergo more rapid tumbling, pointing to an increase in the entropy of water; this finding indicated that the entropic recoil of hydrated elastin does not arise from a change in water entropy ${ }^{32}$. In a separate study, we investigated the effects of glucose on the elastic recoil of elastin through a combined experimental and computational approach ${ }^{24,64}$. In glucose, elastin is 
found to exhibit stiffer characteristics as well as viscoelastic behavior. Our computational results showed that the changes in elasticity arise from a glucose-peptide interaction in motifs such as VPGVG, making the energetic contribution $\left(f_{e}\right)$ non-negligible in the glucose solution. Additionally, we have previously shown through molecular dynamics simulations on an elastin repeat LGGVG, that the retractive forces due to changes in entropy were similar to VPGVG $^{65}$.

We performed molecular dynamic simulations on the elastin repeated $[\mathrm{VPGVG}]_{5}$ motif to provide insight into the changes in the entropic and energetic forces after cholesterol treatment. The peptide entropy can be predicted based on atomic displacements by the quasiharmonic approach. The entropy of the system can be calculated using the expression ${ }^{66}$

$$
S=k_{B} \sum_{i}^{3 n-6} \frac{\hbar \omega_{i} / k_{B} T}{\mathrm{e}^{\hbar \omega_{i} / k_{B} T}-1}-\ln \left(1-\mathrm{e}^{-\hbar \omega_{i} / k_{B} T}\right)
$$

where $\omega_{i}$ are determined from the eigenvalues of the mass weighted covariance matrix of the coordinate fluctuations $\sigma, \sigma^{\prime}=M^{1 / 2} \sigma M^{1 / 2}$, and the sum is over all nonzero eigenvalues. The determination of the entropy also provides a method to measure the equilibration of simulations. Thus, simulations are performed until the entropy reaches an asymptotic value. In the supplementary materials Fig. S1 we show the converged entropy determined from Eq. 9 versus simulation time for the model peptide in either cholesterol solution or in water.

Table 5 shows physical characteristics of the peptides in water and in a $1 \%$ cholesterol solution. We note changes in the radius of gyration following cholesterol exposure which alters the configurational entropy and the entropic force $\left(f_{s}\right)$. Table 6 shows the entropic, energetic, and total forces from accessible nonbonded interactions for VPGVG in both water and cholesterol solution. The cholesterol treated peptide exhibits a smaller entropic force than when the peptide is only in water. Following cholesterol exposure the total retractive force of the repeat VPGVG reduces from 8.0 to $5.3 \mathrm{~kJ} /(\mathrm{mol} \AA)$, and may be a significant contribution to the reduction in stiffness in the cholesterol-treated sample.

The Ramachandran maps for the untreated and cholesterol treated peptides show some 
subtle changes in the proline $(\psi, \phi)$ and valine $(\psi, \phi)$ dihedral angles; shown are the dihedral angles for the two valine and the proline residues in the centroid pentamer of $\mathrm{VPGVG}_{5}$ in Fig. S2. For both valine and proline residues the simulations indicate that the angular distribution $\psi$ is slightly larger for the peptide in cholesterol, in comparison to the peptide in water. This points to an extended structure which may be responsible for the alterations in the the peptide-water interactions (the number of hydrogen bonds with water reduces from $34 \pm 3$ to $23 \pm 3$ for the peptide in a relaxed state in water compared to cholesterol, respectively) which are given in Table 5. The changes in the amount of water in proximity to the peptide also reduces from for the peptide in cholesterol, which alters its dynamical characteristics. Supplementary Fig. S3 shows a histogram of the frequency distribution for the model peptide in either cholesterol or in water; in cholesterol the population of high frequency modes are slightly larger and reduce the entropy (high frequency terms contribute less to entropy than low frequency modulations). These dynamical changes contribute to the entropy in Eq. 9 and alter the entropic retractive force of the peptide. 
Table 5: Simulated retractive forces for $[\text { VGPVG] }]_{5}$ under strained and unstrained (relaxed) conditions, in either $1 \%$ by volume cholesterol or water (details regarding the water model are provided in the Materials and Methods section). In the table, the radius of gyration $(\mathrm{Rg})$ is the distance between the center of gravity of a peptide and its ends. Also shown are the accessible non-bonded energies between the peptides and water or glucose and the intrapeptide energy. The Coulomb potential includes a cut-off distance set to $0.9 \mathrm{~nm}$ and the long-range Coulomb energies are not accessible. The RMSF is the root mean-square fluctuation only in the $\mathrm{C}_{\alpha}$ carbons of the peptides. Finally, we list the number of water molecules within 0.30 $\mathrm{nm}$ of the peptides and the number of hydrogen bonds for the peptide with water and with glucose. The radii of gyration, the RMSF values, and the numbers of water molecules are computed from data in the final $1 \mathrm{~ns}$ of the simulation. The determination of the entropy, using the quasi harmonic approach, made use of 4 ns (or 60 ns for relaxed simulations only) of simulation time with the entropy using the whole window reported.

\begin{tabular}{|c|c|c|c|c|c|c|c|c|c|}
\hline & $\begin{array}{l}\mathrm{Rg} \\
{[\mathrm{nm}]}\end{array}$ & $\begin{array}{l}\text { Water- } \\
\text { Peptide } \\
\text { Energy } \\
{[\mathrm{kJ} / \mathrm{mol}]}\end{array}$ & $\begin{array}{l}\text { Peptide- } \\
\text { Peptide } \\
\text { Energy } \\
{[\mathrm{kJ} / \mathrm{mol}]}\end{array}$ & $\begin{array}{l}\text { Peptide- } \\
\text { Cholesterol } \\
\text { Energy } \\
{[\mathrm{kJ} / \mathrm{mol}]}\end{array}$ & $\begin{array}{l}\text { Entropy } \\
{[\mathrm{J} /(\mathrm{mol} \mathrm{K})]}\end{array}$ & $\begin{array}{l}\text { RMSF } \\
{[\mathrm{nm}]}\end{array}$ & $\begin{array}{l}\text { Number } \\
\text { of water } \\
\text { molecules } \\
\text { within } \\
0.30 \mathrm{~nm} \text { of } \\
\text { peptide }\end{array}$ & $\begin{array}{l}\text { Number of } \\
\text { hydrogen } \\
\text { bonds } \\
\text { with water }\end{array}$ & $\begin{array}{l}\text { Number of } \\
\text { hydrogen } \\
\text { bonds } \\
\text { with } \\
\text { cholesterol }\end{array}$ \\
\hline $\begin{array}{l}\text { cholesterol } \\
\text { (strained) }\end{array}$ & $\begin{array}{l}2.985 \pm \\
0.004 \\
\end{array}$ & -1687 & -314 & -102 & 1015 & $\begin{array}{l}0.023 \pm \\
0.004\end{array}$ & $50 \pm 4$ & $31 \pm 4$ & 0 \\
\hline $\begin{array}{l}\begin{array}{l}\text { cholesterol } \\
\text { (relaxed) }\end{array} \\
\end{array}$ & $\begin{array}{ll}0.75 & \pm \\
0.01 & \\
\end{array}$ & $\begin{array}{l}-974 \\
\end{array}$ & -904 & -235 & 3264 & $\begin{array}{ll}0.12 & \pm \\
0.05 & \\
\end{array}$ & $108 \pm 12$ & $23 \pm 3$ & 0 \\
\hline $\begin{array}{l}\text { water } \\
\text { (strained) }\end{array}$ & $\begin{array}{l}2.986 \pm \\
0.004\end{array}$ & -1756 & -314 & - & 1152 & $\begin{array}{l}0.025 \pm \\
0.003\end{array}$ & $234 \pm 14$ & $48 \pm 4$ & - \\
\hline $\begin{array}{l}\text { water } \\
\text { (relaxed) }\end{array}$ & $\begin{array}{ll}0.68 & \pm \\
0.01 & \\
\end{array}$ & -1114 & -939 & - & 3500 & $\begin{array}{ll}0.50 & \pm \\
0.21 & \\
\end{array}$ & $134 \pm 13$ & $34 \pm 3$ & - \\
\hline
\end{tabular}

Table 6: Simulation results for the retractive forces of a $[\text { VGPVG] }]_{5}$ peptide. The entropic force and energy forces are computed from values shown in Table 5. The entropic force is given by $f_{s}=-T \Delta s /(\Delta r)(T=310 \mathrm{~K})$ and the energy force is given by $\mathrm{f}_{e}=\Delta U / \Delta r$.

\begin{tabular}{|l|l|l|l|l|}
\hline & $\begin{array}{l}\text { End to end dis- } \\
\text { placement } \Delta \mathrm{r}[\AA]\end{array}$ & $\begin{array}{l}\text { Entropic Force } \\
{[\mathrm{kJ} /(\mathrm{mol} \AA)]}\end{array}$ & $\begin{array}{l}\text { Energy Force } \\
{[\mathrm{kJ} /(\mathrm{mol} \AA)]}\end{array}$ & $\begin{array}{l}\text { Total Force } \\
{[\mathrm{kJ} /(\mathrm{mol} \AA)]}\end{array}$ \\
\hline water & 89.4 & 8.1 & -0.2 & 8.0 \\
\hline cholesterol & 91.7 & 5.2 & 0.1 & 5.3 \\
\hline
\end{tabular}




\section{Conclusions}

We have reported macroscopic mechanical behavior and microscopic dynamical and structural modifications of porcine aortic elastin after cholesterol exposure. Mechanical measurements indicate that the cholesterol treated elastin sample exhibits a decrease in stiffness. The dimensional and mechanical changes of elastin also suggest that the cholesterol treatment may cause microstructural changes in the elastin matrix. Our ${ }^{13} \mathrm{C}$ MAS NMR spectra show that the cholesterol treated sample cross polarizes less than the untreated sample, indicating an increase in mobility following cholesterol exposure. These findings were further corroborated by ${ }^{13} \mathrm{C}$ NMR relaxation measurements which showed a reduction in the ${ }^{1} \mathrm{H}_{-}{ }^{13} \mathrm{C}$ internuclear vector correlation times following cholesterol exposure. Two-dimensional ${ }^{2} \mathrm{H} T_{1^{-}}$ $T_{2}$ NMR techniques allowed a measurement of the dynamics and distribution of water in the untreated and cholesterol treated samples. Cholesterol treatment led to a redistribution of water with changes in the population of water in the most mobile reservoir. Molecular dynamics simulations on the motif VPGVG in cholesterol and water were performed to quantify

changes in peptide-solvent and peptide-peptide hydrogen bonds, dynamics, as well as the entropic and energetic changes. Exposure to cholesterol is observed to alter the dynamics and conformation of this short elastin repeat which in turn reduces the retractive entropic force, and may play a significant role in the observed mechanical behavior of cholesterol exposed elastin.

\section{Acknowledgements}

The authors thank Boris Itin, Antony Papaioannou, and Ivan Sergeyev for assistance in the NMR experiments and for useful discussions and Yi- Qiao Song of Schlumberger-Doll Research for allowing us to implement the ILT algorithm. G.S.B. is a member of the New York Structural Biology Center.

G.S.B. acknowledges support from the National Institutes of Health under award number 
2SC1GM086268. Y. Z. acknowledges support from Kenneth R. Lutchen Distinguished Fellowship from the College of Engineering, Student Research Award from the Undergraduate Research Opportunities Program (UROP) at Boston University, National Science Foundation grants CMMI 1100791 (Research Experiences for Undergraduates) and CMMI 1463390. Collection of data at the New York Structural Biology Center was made possible by a grant from NYSTAR. This research was supported, in part, by a grant of computer time from the City University of New York High Performance Computing Center under National Science Foundation grants CNS-0855217, CNS-0958379, and ACI-1126113.

\section{References}

(1) Kielty, C. M.; Sherratt, M. J.; Shuttleworth, C. A. Elastic fibres. J. Cell Sci. 2002, $115,2817-2828$.

(2) Zou, Y.; Zhang, Y. An experimental and theoretical study on the anisotropy of elastin network. Ann. Biomed. Eng. 2009, 37, 1572-1583.

(3) Gosline, J.; Lillie, M.; Carrington, E.; Guerette, P.; Ortlepp, C.; Savage, K. Elastic proteins: biological roles and mechanical properties. Philos. T. Roy. Soc. B 2002, 35\%, $121-132$.

(4) Lillie, M.; Gosline, J. Effects of lipids on elastin's viscoelastic properties. Biopolymers 2002, 64, 127-138. 
(5) McGrath, L. T.; Elliott, R. J. Formation of a lipid gradient across the human aortic wall during ageing and the development of atherosclerosis. Atherosclerosis 1991, 87, $211-220$.

(6) Saulnier, J. M.; Hauck, M.; Fülöp, T.; Wallach, J. M. Human aortic elastin from normal individuals and atherosclerotic patients: lipid and cation contents; susceptibility to elastolysis. Clin. Chim. Acta 1991, 200, 129-136.

(7) Mackey, R.; Venkitachalam, L.; Sutton-Tyrrell, K. Calcifications, arterial stiffness and atherosclerosis. Atherosclerosis, Large Arteries and Cardiovascular Risk 44, 234-244.

(8) van Popele, N. M.; Grobbee, D. E.; Bots, M. L.; Asmar, R.; Topouchian, J.; Reneman, R. S.; Hoeks, A. P.; van der Kuip, D. A.; Hofman, A.; Witteman, J. C. Association between arterial stiffness and atherosclerosis The Rotterdam Study. Stroke 2001, 32, 454-460.

(9) Kramsch, D. M.; Hollander, W. The interaction of serum and arterial lipoproteins with elastin of the arterial intima and its role in the lipid accumulation in atherosclerotic plaques. Journal of Clinical Investigation 1973, 52, 236.

(10) Kramsch, D. M.; Franzblau, C.; Hollander, W. The protein and lipid composition of arterial elastin and its relationship to lipid accumulation in the atherosclerotic plaque. Journal of Clinical Investigation 1971, 50, 1666.

(11) Tarnawski, R.; Tarnawski, R.; Grobelny, J. Changes in elastin in human atherosclerotic aorta: Carbon-13 magic angle sample-spinning NMR studies. Atherosclerosis 1995, $115,27-33$.

(12) Wilkinson, I.; Cockcroft, J. Cholesterol, lipids and arterial stiffness. Atherosclerosis, Large Arteries and Cardiovascular Risk 2007, 44, 261-277. 
(13) Rosenbloom, J.; Abrams, W.; Mecham, R. Extracellular matrix 4: the elastic fiber. FASEB J. 1993, \%, 1208-1218.

(14) Debelle, L.; Tamburro, A. Elastin: molecular description and function. Int. J. Biochem. Cell Biol. 1999, 31, 261-272.

(15) He, D.; Chung, M.; Chan, E.; Alleyne, T.; Ha, K. C.; Miao, M.; Stahl, R. J.; Keeley, F. W.; Parkinson, J. Comparative genomics of elastin: Sequence analysis of a highly repetitive protein. Matrix Biol. 2007, 26, 524-540.

(16) Luan, C.-H.; Jaggard, J.; Harris, R. D.; Urry, D. W. On the source of entropic elastomeric force in polypeptides and proteins: Backbone configurational vs. side-chain solvational entropy. Int. J. Quantum Chem. 1989, 36, 235-244.

(17) Yao, X.; Hong, M. Structure distribution in an elastin-mimetic peptide (VPGVG) 3 investigated by solid-state NMR. J. Amer. Chem. Soc 2004, 126, 4199-4210.

(18) Yao, X. L.; Conticello, V. P.; Hong, M. Investigation of the dynamics of an elastinmimetic polypeptide using solid-state NMR. Magn. Reson. Chem. 2004, 42, 267-275.

(19) Torchia, D.; Piez, K. Mobility of elastin chains as determined by $13 \mathrm{C}$ nuclear magnetic resonance. J. Mol. Biol. 1973, 76, 419-424.

(20) Lyerla Jr, J.; Torchia, D. Molecular mobility and structure of elastin deduced from the solvent and temperature dependence of carbon-13 magnetic resonance relaxation data. Biochemistry 1975, 14, 5175-5183.

(21) Kumashiro, K. K.; Kim, M. S.; Kaczmarek, S. E.; Sandberg, L. B.; Boyd, C. D. 13C cross-polarization/magic angle spinning NMR studies of $\alpha$-elastin preparations show retention of overall structure and reduction of mobility with a decreased number of cross-links. Biopolymers 2001, 59, 266-275. 
(22) Demuth, D.; Haase, N.; Malzacher, D.; Vogel, M. Effects of solvent concentration and composition on protein dynamics: 13 C MAS NMR studies of elastin in glycerol-water mixtures. Biochim. Biophys. Acta, Proteins Proteomics 2015, 1854, 995-1000.

(23) Lillie, M.; Gosline, J. Swelling and viscoelastic properties of osmotically stressed elastin. Biopolymers 1996, 39, 641-652.

(24) Silverstein, M. C.; Bilici, K.; Morgan, S. W.; Wang, Y.; Zhang, Y.; Boutis, G. S. 13 C, 2 H NMR Studies of Structural and Dynamical Modifications of Glucose-Exposed Porcine Aortic Elastin. Biophys. J. 2015, 108, 1758-1772.

(25) Sahni, D.; Kaur, G.; Jit, H.; Jit, I. Anatomy \&amp; distribution of coronary arteries in pig in comparison with man. Indian J. Med. Res. 2008, 127, 564.

(26) Lu, Q.; Ganesan, K.; Simionescu, D. T.; Vyavahare, N. R. Novel porous aortic elastin and collagen scaffolds for tissue engineering. Biomaterials 2004, 25, 5227-5237.

(27) Hornebeck, W.; Partridge, S. M. Conformational changes in fibrous elastin due to calcium ions. Eur. J. Biochem. 1975, 51, 73-78.

(28) Zou, Y.; Zhang, Y. The orthotropic viscoelastic behavior of aortic elastin. Biomech. Model. Mechanobiol. 2011, 10, 613-625.

(29) Chow, M.-J.; Zhang, Y. Changes in the mechanical and biochemical properties of aortic tissue due to cold storage. J. Surg. Res. 2011, 171, 434-442.

(30) Chow, M.-J.; Mondonedo, J. R.; Johnson, V. M.; Zhang, Y. Progressive structural and biomechanical changes in elastin degraded aorta. Biomech. Model. Mechanobiol. 2013, 12, 361-372.

(31) Bennett, A. E.; Rienstra, C. M.; Auger, M.; Lakshmi, K.; Griffin, R. G. Heteronuclear decoupling in rotating solids. J. Chem. Phys. 1995, 103, 6951-6958. 
(32) Sun, C.; Mitchell, O.; Huang, J.; Boutis, G. S. NMR studies of localized water and protein backbone dynamics in mechanically strained elastin. J. Phys. Chem. B 2011, $115,13935-13942$.

(33) Song, Y.-Q.; Venkataramanan, L.; Hürlimann, M.; Flaum, M.; Frulla, P.; Straley, C. T 1-T 2 correlation spectra obtained using a fast two-dimensional Laplace inversion. $J$. Magn. Reson. 2002, 154, 261-268.

(34) Pronk, S.; Pall, S.; Schulz, R.; Larsson, P.; Bjelkmar, P.; Apostolov, R.; Shirts, M. R.; Smith, J. C.; Kasson, P. M.; van der Spoel, D.; Hess, B.; Lindahl, E. GROMACS 4.5: a high-throughput and highly parallel open source molecular simulation toolkit. Bioinformatics 2013, 29, 845-854.

(35) Oostenbrink, C.; Villa, A.; Mark, A. E.; Van Gunsteren, W. F. A biomolecular force field based on the free enthalpy of hydration and solvation: The GROMOS force-field parameter sets 53A5 and 53A6. J. Comput. Chem. 2004, 25, 1656-1676.

(36) Teleman, O.; Jönsson, B.; Engström, S. A molecular dynamics simulation of a water model with intramolecular degrees of freedom. Mol. Phys. 1987, 60, 193-203.

(37) Bussi, G.; Donadio, D.; Parrinello, M. Canonical sampling through velocity rescaling. J. Chem. Phys. 2007, 126, 014101.

(38) Papaioannou, A.; Louis, M.; Dhital, B.; Ho, H.; Chang, E.; Boutis, G. Quantitative comparison of structure and dynamics of elastin following three isolation schemes by 13 C solid state NMR and MALDI mass spectrometry. Biochim. Biophys. Acta, Proteins Proteomics 2015, 1854, 391-401.

(39) Pometun, M. S.; Chekmenev, E. Y.; Wittebort, R. J. Quantitative observation of backbone disorder in native elastin. J. Biol. Chem. 2004, 279, 7982-7987. 
(40) Kumashiro, K. K.; Ho, J. P.; Niemczura, W. P.; Keeley, F. W. Cooperativity between the hydrophobic and cross-linking domains of elastin. J. Biol. Chem. 2006, 281, 2375723765 .

(41) Ijare, O. B.; Somashekar, B.; Jadegoud, Y.; Gowda, G. N. 1H and 13C NMR characterization and stereochemical assignments of bile acids in aqueous media. Lipids 2005, 40, 1031-1041.

(42) Chang, D.; Urry, D. Polypentapeptide of elastin: damping of internal chain dynamics on extension. J. Comput. Chem. 1989, 10, 850-855.

(43) Urry, D.; Parker, T. Mechanics of Elastic Biomolecules; Springer, 2003; pp 543-559.

(44) Sun, C.; Boutis, G. S. Measurement of the exchange rate of waters of hydration in elastin by 2D T2-T2 correlation nuclear magnetic resonance spectroscopy. New J. Phys 2011, 13, 025026 .

(45) Perry, A.; Stypa, M. P.; Tenn, B. K.; Kumashiro, K. K. Solid-State 13 C NMR Reveals Effects of Temperature and Hydrationon Elastin. Biophysical journal 2002, 82, 10861095.

(46) Duer, M. J. Solid state NMR spectroscopy: principles and applications; John Wiley \& Sons, 2008.

(47) Asakura, T.; Iwadate, M.; Demura, M.; Williamson, M. P. Structural analysis of silk with 13 C NMR chemical shift contour plots. Int. J. Biol. Macromol. 1999, 24, 167-171.

(48) Wang, Y.; Jardetzky, O. Probability-based protein secondary structure identification using combined NMR chemical-shift data. Protein Sci. 2002, 11, 852-861.

(49) Zhang, H.; Neal, S.; Wishart, D. S. RefDB: a database of uniformly referenced protein chemical shifts. J. Biomol. NMR 2003, 25, 173-195. 
(50) Jenkins, J. E.; Creager, M. S.; Lewis, R. V.; Holland, G. P.; Yarger, J. L. Quantitative correlation between the protein primary sequences and secondary structures in spider dragline silks. Biomacromolecules 2009, 11, 192-200.

(51) Wishart, D. S.; Bigam, C. G.; Holm, A.; Hodges, R. S.; Sykes, B. D. 1H, 13C and $15 \mathrm{~N}$ random coil NMR chemical shifts of the common amino acids. I. Investigations of nearest-neighbor effects. J. Biomol. NMR 1995, 5, 67-81.

(52) Wishart, D. S.; Bigam, C. G.; Yao, J.; Abildgaard, F.; Dyson, H. J.; Oldfield, E.; Markley, J. L.; Sykes, B. D. 1H, 13C and 15N chemical shift referencing in biomolecular NMR. J. Biomol. NMR 1995, 6, 135-140.

(53) Quinn, C. M.; McDermott, A. E. Monitoring conformational dynamics with solid-state R $1 \rho$ experiments. J. Biomol. NMR 2009, 45, 5-8.

(54) Kurbanov, R.; Zinkevich, T.; Krushelnitsky, A. The nuclear magnetic resonance relaxation data analysis in solids: General R1/R1 $\rho$ equations and the model-free approach. J. Chem. Phys. 2011, 135, 184104.

(55) Miao, M.; Bellingham, C. M.; Stahl, R. J.; Sitarz, E. E.; Lane, C. J.; Keeley, F. W. Sequence and structure determinants for the self-aggregation of recombinant polypeptides modeled after human elastin. J. Biol. Chem. 2003, 278, 48553-48562.

(56) Bellingham, C. M.; Lillie, M. A.; Gosline, J. M.; Wright, G. M.; Starcher, B. C.; Bailey, A. J.; Woodhouse, K. A.; Keeley, F. W. Recombinant human elastin polypeptides self-assemble into biomaterials with elastin-like properties. Biopolymers 2003, 70, 445455 .

(57) Song, Y.-Q. Novel NMR techniques for porous media research. J. Magn. Reson. Imaging 2003, 21, 207-211. 
(58) Lang, E.; Lüdemann, H.-D.; Piculell, L. Nuclear magnetic relaxation rate dispersion in supercooled heavy water under high pressure. J. Chem. Phys. 1984, 81, 3820-3827.

(59) Weis-Fogh, T.; Andersen, S. New molecular model for the long-range elasticity of elastin. Nature 1970, 227, 718-721.

(60) Ukpebor, O. T.; Shah, A.; Bazov, E.; Boutis, G. S. Inverse temperature transition of elastin like motifs in major ampullate dragline silk: MD simulations of short peptides and NMR studies of water dynamics. Soft matter 2014, 10, 773-785.

(61) Flory, P.; Ciferri, A.; Hoeve, C. The thermodynamic analysis of thermoelastic measurements on high elastic materials. J. Polym. Sci. 1960, 45, 235-236.

(62) Tatham, A. S.; Shewry, P. R. Elastomeric proteins: biological roles, structures and mechanisms. Trends Biochem. Sci. 2000, 25, 567-571.

(63) Wasserman, Z; Salemme, F. A molecular dynamics investigation of the elastomeric restoring force in elastin. Biopolymers 1990, 29, 1613-1631.

(64) Wang, Y.; Zeinali-Davarani, S.; Davis, E. C.; Zhang, Y. Effect of glucose on the biomechanical function of arterial elastin. J. Mech. Behav. Biomed. Mater 2015, 49, 244-254.

(65) Huang, J.; Sun, C.; Mitchell, O.; Ng, N.; Wang, Z. N.; Boutis, G. S. On the inverse temperature transition and development of an entropic elastomeric force of the elastin mimetic peptide [LGGVG] 3, 7. J. Chem. Phys 2012, 136, 085101.

(66) Andricioaei, I.; Karplus, M. On the calculation of entropy from covariance matrices of the atomic fluctuations. J. Chem. Phys. 2001, 115, 6289-6292. 